\title{
Pelvic actinomycosis: unrelated to prior intrauterine device use
}

\author{
Emily Divya Ebenezer*, Vaibhav Londhe, Lilly Varghese, Mayank Gupta, Aruna N. Kekre
}

Department of Obstetrics and Gynaecology, Christian Medical College, Vellore, India

Received: 13 January 2016

Revised: 14 February 2016

Accepted: 16 February 2016

\author{
*Correspondence: \\ Dr. Emily Divya Ebenezer, \\ E-mail: emilydivya@gmail.com
}

Copyright: () the author(s), publisher and licensee Medip Academy. This is an open-access article distributed under the terms of the Creative Commons Attribution Non-Commercial License, which permits unrestricted non-commercial use, distribution, and reproduction in any medium, provided the original work is properly cited.

\begin{abstract}
Actinomycosis is a chronic, suppurative, granulomatous disease which is typically seen in the cervicofacial and thoracic regions and less commonly abdominopelvic (10-20\%). It is caused by an anaerobic, filamentous gram positive bacterium which is a normal commensal of the oropharynx, gastrointestinal tract and genital tract. Pelvic actinomycosis is almost always associated with the current or prior use of an intrauterine contraceptive device. Clinical diagnosis is difficult owing to the close resemblance to an ovarian tumour. Definitive diagnosis is usually made only after histopathological correlation. Reported here is a patient with pelvic actinomycosis unrelated to the use of an intrauterine contraceptive device. This patient presented with clinical features suggestive of chronic pelvic inflammatory disease with a possible benign ovarian tumour. Operative findings were consistent with pelvic inflammatory disease. The typical histopathological features of the Splendor Hoeppli phenomenon with sulphur granules and isolation of the actinomyces organism (which is rare) revealed the diagnosis of actinomycosis of the fallopian tubes. Pelvic actinomycosis is a rare condition and seldom described in the absence of an intrauterine device. High dose, long term antibiotic therapy is the key to successful resolution with surgery reserved for refractory cases. This patient showed good clinical and radiological response.
\end{abstract}

Keywords: Pelvic actinomycosis, IUCD, Pelvic inflammatory disease, Actinomycosis fallopian tubes, Sulphur granules, Splender Hoeppli phenomenon, High dose penicillin

\section{INTRODUCTION}

Actinomycosis is a chronic, suppurative, granulomatous disease which is typically seen in the cervicofacial and thoracic regions and less commonly abdominopelvic (10$20 \%){ }^{1}$ It is caused by an anaerobic, filamentous gram positive bacterium which is a normal commensal of the oropharynx, gastrointestinal tract and genital tract. Pelvic actinomycosis is almost always associated with the current or prior use of an intrauterine contraceptive device. ${ }^{2}$ Clinical diagnosis is difficult owing to the close resemblance to an ovarian tumour. ${ }^{3}$ Definitive diagnosis is usually made only after histopathological correlation. ${ }^{4}$ Reported here is a patient with pelvic actinomycosis unrelated to the use of an intrauterine contraceptive device. A brief review of relevant literature is also presented.

\section{CASE REPORT}

A 29 year old lady presented with complaints of lower abdominal pain and yellowish discharge per vaginum on and off for a period of one month. She had a background of primary infertility for 8 years and on evaluation for the same, had been diagnosed to have chronic pelvic inflammatory disease. She had undergone a laparotomy and excision of a left ovarian haemorrhagic cyst 12 years earlier. She had no history of having had an intrauterine device at any time.

Clinical examination revealed a large, $10 \times 12 \mathrm{~cm}$, right sided abdominopelvic mass with ill-defined borders and restricted mobility. There was no ascites or lymphadenopathy and the rest of the clinical examination was unremarkable. 
Imaging (ultrasound abdomen) showed a 16 × $10 \mathrm{~cm}$ multiloculated cyst in the pelvis with thick septations and internal echoes, however there were no solid areas or papillary excresences. The ovaries could not be visualised separately and there was no ascites. Tumour markers were in the normal range. With the working diagnosis of a probable ovarian tumour, she was planned for a laparotomy.

Operative findings revealed dense pelvic and peritubal adhesions, consistent with a chronic inflammatory process. A right adnexal cyst of $15 \times 10 \mathrm{~cm}$ with clear fluid was present along with thickened and dilated fallopian tubes filled with purulent material. Adhesiolysis and bilateral adnexectomy was performed. The histopathology showed sections of the fallopian tube with moderate to dense infiltrates of neutrophils, lymphocytes, eosinophils and foamy histiocytes with inflammatory exudates in the lumen. The classic appearance of sulphur granules characterized by the Splendor- Hoeppli phenomenon with a peripheral eosinophilic rim and a basophilic center was present. The periphery of the granule showed gram positive, branching, filamentous bacteria which were positive for Periodic Acid Schiff (PAS) and Grocott Methenamine Silver (GMS) stain, typical of actinomyces.

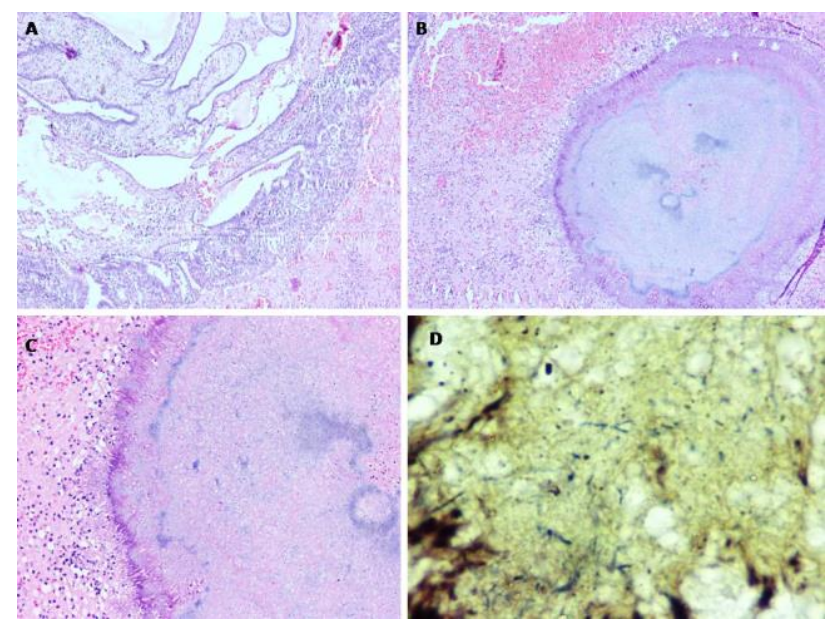

Figure 1: A; Hematoxylin and eosin (H\&E) stain showing wall of fallopian tube with subepithelial chronic inflammation, 10x magnification; $\mathrm{B}$; H\&E stain showing a granule displaying central basophilia and a peripheral rim of eosinophilic, club-like projections composed of immunoglobulin, fibrin, and tissue debris (Splendore-Hoeppli phenomenon), 10x magnification; $\mathrm{C}$; H\&E stain displaying granule at

20x magnification; D; Grams stain showing gram positive filamentous bacilli, 100x magnification. Acid fast stain was negative.

Hence, she was given high dose parenteral penicillin for 2 weeks and subsequently put on oral amoxicillin for a total of 3 months. She showed good clinical and radiological response.
Repeat ultrasonography done after 2 months of treatment showed no features of residual pelvic inflammatory disease, with a normal uterus and cavity and no pelvic masses.

\section{DISCUSSION}

Pelvic actinomycosis is a rare condition with only a few cases reported in literature. ${ }^{5} 85-90 \%$ of these have been associated with the use of an intrauterine device. ${ }^{6-10}$ The proposed pathogenesis in those with an IUD is the development of a biofilm on the copper surface and subsequent invasion of breached tissue. ${ }^{2,11,12}$ Here we report a patient with pelvic actinomycosis unrelated to the use of an IUCD.

A few cases have been reported of pelvic actinomycosis unrelated to the use of an IUD. Interestingly, each of these patients have had a prior pelvic procedure, one after hysteroscopic coil sterilization, two patients after mesh surgeries for stress incontinence, and one patient after transvaginal oocyte retrieval. ${ }^{13-15}$ The patient reported here had chronic pelvic inflammatory disease and a previous surgery for removal of a tubo-ovarian mass.

Coinfection of actinomyces along with more common causative organisms for PID, like Neisseria gonorrhoea, has been described. ${ }^{10,16,17}$

Definitive clinical diagnosis of this condition is difficult since it usually mimics an ovarian tumour. ${ }^{6-8}$ More often than not, it is the pathologist who first diagnoses this condition after extensive surgery. ${ }^{18}$ It may be worthwhile to keep this in mind as a differential when dealing with young patients with an adnexal cyst with no other features of malignancy and a history of longstanding Copper-T use.

CT and ultrasound guided aspirations of the abscesses have sometimes yielded positive results. ${ }^{18,19}$

Isolation of the organism however, is challenging, and microbiological identification occurs only in a minority. ${ }^{20}$ This has been attributed to the fragility of the germ and its extreme sensitivity to common antibiotics thereby reducing the final yield available for culture. .,12,21 $^{2}$

The most definite method of diagnosis is the demonstration of the presence of macroscopic sulphur granules (which is a conglomeration of the organisms) or histology showing microscopic granules with the organism on special staining.

Due to the rarity of the disease, treatment is based on clinical experience as there are no randomized controlled trials. High dose, long term antibiotic therapies are the key principles to successful resolution. ${ }^{21-23}$ Protracted treatment is required for extensive disease. Duration of antibiotic therapy should be individualized based on clinical response. As medical therapy is efficacious, 
surgery may be reserved for extensive and refractory disease. $^{24}$

Funding: No funding sources

Conflict of interest: None declared

Ethical approval: Not required

\section{REFERENCES}

1. Könönen E, Wade WG. Actinomyces and related organisms in human infections. Clin Microbiol Rev. 2015;28:419-42.

2. Westhoff C. IUDs and colonization or infection with Actinomyces. Contraception. 2007;75:S48-50.

3. Hildyard CAT, Gallacher NJ, Macklin PS. Abdominopelvic actinomycosis mimicking disseminated peritoneal carcinomatosis. BMJ Case Rep. 2013.

4. Wong VK, Turmezei TD, Weston VC. Actinomycosis. BMJ. 2011;343:d6099.

5. Marret H, Wagner N, Ouldamer L, Jacquet A. Body, G. Pelvic actinomycosis: just think of it. Gynécologie Obstétrique Fertil. 2010;38:307-12.

6. Abid M. Intrauterine device and pelvic tumor: two case reports of pelvic actinomycosis with pseudotumor from tropical zones. Médecine Trop. Rev. Corps Santé Colon. 2010;70:285-7.

7. Elhassani ME. Pseudo tumor pelvic actinomycosis associated with intrauterine device: report of three cases. Pan Afr Med J. 2014;19:87.

8. Morland D, Hassler S. Case 219: Pelvic Actinomycosis Mimicking Malignant Tumor. Radiology. 2015;276:304-8.

9. Chelli D. Pelvic actinomycosis in Tunisia: five cases. Santé Montrouge Fr. 2008;18:77-82.

10. Kim YJ, Youm J, Kim JH, Jee BC. Actinomyces-like organisms in cervical smears: the association with intrauterine device and pelvic inflammatory diseases. Obstet Gynecol Sci. 2014;57:393-6.

11. Shanmughapriya S, Francis AL, Kavitha S, Natarajaseenivasan K. In vitro actinomycete biofilm development and inhibition by the polyene antibiotic, nystatin, on IUD copper surfaces. Biofouling. 2012;28:929-35.

12. Carrillo M. In vitro Actinomyces israelii biofilm development on IUD copper surfaces. Contraception. 2010;81:261-4.
13. Masata J, Dundr P, Martan A. Actinomyces infection appearing five years after trocar-guided transvaginal mesh prolapse repair. Int Urogynecology J. 2014;25:993-6.

14. Ozel B, Kuo J, Minaglia S. Actinomyces infection associated with the transobturator sling. Int Urogynecology J. 2010;21:121-3.

15. Asemota OA, Girda E, Dueñas O, Neal-Perry G, Pollack SE. Actinomycosis pelvic abscess after in vitro fertilization. Fertil Steril. 2013;100:408-11.

16. Eiros-Bouza JM, González MDG, Martín-Medrano E. Co-infection with Neisseria gonorrhoeae and Actinomyces naeslundii. Ginecol. Obstet México. 2013;81:665-7.

17. Arora BB, Maheshwari M, Devgan N, Arora DR. Prevalence of Trichomoniasis, Vaginal Candidiasis, Genital Herpes, Chlamydiasis, and Actinomycosis among Urban and Rural Women of Haryana, India. J Sex Transm Dis. 2014;963812.

18. Pauker SG, Kopelman RI. Clinical problem-solving. A rewarding pursuit of certainty. $\mathrm{N}$ Engl $\mathrm{J}$ Med. 1993;329:1103-7.

19. Chelly I. Renal actinomycosis: a case report. Médecine Trop Rev Corps Santé Colon. 2006;66:266-8.

20. Brown JR. Human actinomycosis. A study of 181 subjects. Hum. Pathol. 1973;4:319-30.

21. Bennhoff DF. Actinomycosis: diagnostic and therapeutic considerations and a review of 32 cases. The Laryngoscope. 1984;94:1198-217.

22. Taga S. Diagnosis and therapy of pelvic actinomycosis. J Obstet Gynaecol Res. 2007;33:8825.

23. Atad J. Pelvic actinomycosis. Is long-term antibiotic therapy necessary? J Reprod Med. 1999;44:939-44.

24. Mendel's Infectious Diseases- 7th edition.

Cite this article as: Ebenezer ED, Londhe V, Varghese L, Gupta M, Kekre AN. Pelvic actinomycosis: unrelated to prior intrauterine device use. Int J Reprod Contracept Obstet Gynecol 2016;5:921-3. 